\title{
A Synchronous CAD/CAE Modeling Method and Applications in Parametric Parts Library
}

\author{
Zhiyi Pan ${ }^{*}$, Xin Wang, Rumin Teng and Shunde Gao
}

School of Mechanical Engineering, Dalian University of Technology, Dalian, Liaoning, 116024, P.R. China

\begin{abstract}
Current parametric parts library cannot contribute to the reuse of finite element model. It is essential to remodel or exchange model for parts in CAE software. This paper presents a synchronous CAD/CAE modeling method, which can be employed in parts library based on Siemens NX software. Primary CAD and CAE information in part are merged into master model, which acts as cornerstone for solid model, FE model and simulation model. Thus, part models from parts library can be directly used into simulation analysis, and parametric amendment and change propagation is sustained. A software package developed for designers is helpful to search and achieve desired part from parts library. Three case studies are carried out to clarify synchronous $\mathrm{CAD} / \mathrm{CAE}$ modeling process and change propagation procedure.
\end{abstract}

Keywords: CAD, CAE, Integration, Parts library, Siemens NX software, Current conversion method.

\section{INTRODUCTION}

Establishing a parametric parts library, which is in line with national and enterprise's standard, is necessary for facilitating the reuse of parts and improving product design efficiency. Nowadays, parametric parts library is set up and employed on the basis of commercial CAD software, such as Siemens NX, Pro/Engineering and Solidworks, etc. In order to improve the efficiency of modeling, many efforts have been made to develop a specified parts library by visual programming language, Application Program Interface and configuration technology [1-3]. It is worthwhile to note that many frequently-used parts need to verify that the design fulfills the requirements and constraints by engineering analysis in some machine design domain, for example, construction machinery. Thus, it is essential to re-model the part in CAE software, implement simulation and revise model iteratively till acceptable result is achieved. It is difficult to avoid much duplicated artificial work. Though some model conversion methods utilizing neutral files (e.g. IGES, STEP and STL) and CAD kernels (e.g. ACIS, ParaSolid) are helpful for acceleration work, information lose, model amendment and connection inconsistencies are still unavoidable. Is it feasible to implement simulation and analysis based on part model directly. CAD/CAE integration is an efficient approach to solve the problem.

For a long time, CAD and CAE are disciplines with separate histories and consequently different conventions, data representations and workflows. For most commercial CAD software, the focus is usually on the two processes which are considered as two independent activities and not well integrated [4]. Though the design and analysis models in essence describe the same object, the models are so

*Address correspondence to this author at the School of Mechanical Engineering, Dalian University of Technology, Dalian, Liaoning, 116024, P.R. China; Tel: 13610850186; E-mail: panzhiyi@dlut.edu.cn different that going back and forth between them is a clear bottleneck within product development cycle. Hence, How to seamlessly integrate CAD and CAE has become one of the important issues for efficient product development.

This paper presents a synchronous $\mathrm{CAD} / \mathrm{CAE}$ modeling method, which can be applied in the parts library based on Siemens NX software. Instead of duplicated FE modeling and fussy model exchange, part models from parts library are directly employed into simulation analysis and engineering drawing due to coincident model. Current research work involved in $\mathrm{CAD} / \mathrm{CAE}$ integration approaches are as follows.

\subsection{Current CAD Model Simplification}

Automated CAD model simplification or idealization plays a vital role in effectively utilizing physics-based simulation during the product realization process. Gao Shuming presented a framework to CAD mesh model simplification based on feature suppression. The approach is capable of removing unnecessary form features from the mesh model without any changes to the rest part [5]. Hamdi Mounir presented an idealization algorithm of CAD models for a simulation by the finite element method [6]. Zhu $\mathrm{H}$. pre-processed the input B-Rep models by suppressing fillets and rounds before the feature recognition. Thus the input BRep models can be simplified without altering the major shapes of primary features [7]. Atul Thakur summarized various model simplification techniques available that are applicable for physics-based simulation applications and presented a broad selection criterion for different classes of engineering problems commonly encountered in practice [8]. Mounir H. presented a novel simplification of the CAD geometry technique, which is a hybrid method based on a combination of the elimination details and merging faces. The reduction of the computing time and the amelioration of the result precision highlight the efficiency of the method [9]. 


\subsection{Current Conversion Method from CAD to CAE}

Since data exchange is central to the implementation of a collaborative environment, much previous research efforts focused on standardizing data and data exchange methodologies. Hamri O. proposed an approach to perform efficient model exchange between CAD and FEA software environments based on standards of exchange and particularly STEP [10]. Gilles Foucault put forward a Mesh Constraint Topology model with automatic adaptation operators aimed at transforming CAD model boundary decomposition into a FE model, featuring only meshrelevant faces, edges and vertices [11]. Hamri O. set up a new approach for CAD/E integration based on the concept of mixed representation, which allows the software architecture to explicitly maintain the links between these models and manipulate them [12]. Shapiro V. examined and compared known mesh-based and mesh-free approaches to $\mathrm{CAD} / \mathrm{E}$ integration, focusing on the basic tasks and components required for building fully integrated engineering applications [13]. Park B. K. came up with a Practical PostAnalysis Model, which is a sharable, neutral format for multidisciplinary FEA [14].

\subsection{Current Middleware Development Approach}

In order to integrate information between $\mathrm{CAD}$ and $\mathrm{CAE}$, middleware development approach is also favored widely. Propagation of changes is also managed by optimization methods and embedded knowledge. Hong-Seok Park presented a framework that performs the integration by using common scripting, programming languages and API [15]. Kenworthy presented a method to perform optimization iterations on CAD models utilizing CAD external analysis tools in the software package iSIGHT-FD [16]. G. P. Gujarathi proposed a $\mathrm{CAD} / \mathrm{E}$ integration method using a common data model (CDM), which is automatically generated by knowledge embedded program code and contain all the required parametric information for both CAD modeling and CAE analysis [17]. Lee S. H. reported a new approach CAD/E-integrated implemented by a feature-based non-manifold modeling system [18]. Besides, the methods also used in an integration environment for the design and analysis of plastic injection [19].

\section{SYNCHRONOUS CAD/CAE MODELING METHOD}

With the rapid development of the technique in modern $\mathrm{CAD} / \mathrm{CAE}$ software, it is currently becoming a trend for commercial CAD/CAE suppliers to offer complete solutions for the industry. In several high-end commercial CAD platforms, such as Siemens NX and Dassault System, it has been implemented that simulation activities are integrated with the CAD modeling environment beyond neutral data file. Thus, it is allowed for designers to carry out the geometric modeling and the automatic mesh generation within one software environment. In synchronous CAD/CAE modeling method presented in this paper, primary CAD and CAE information in part is merged into a single pattern named as master model from scratch. Then, detailed solid model and CAE model are established based on master model. According to Fig. (1), master model acts as cornerstone for solid model, FE model and simulation model.
Chief parameter model can revise some other models in parameterization strategy.

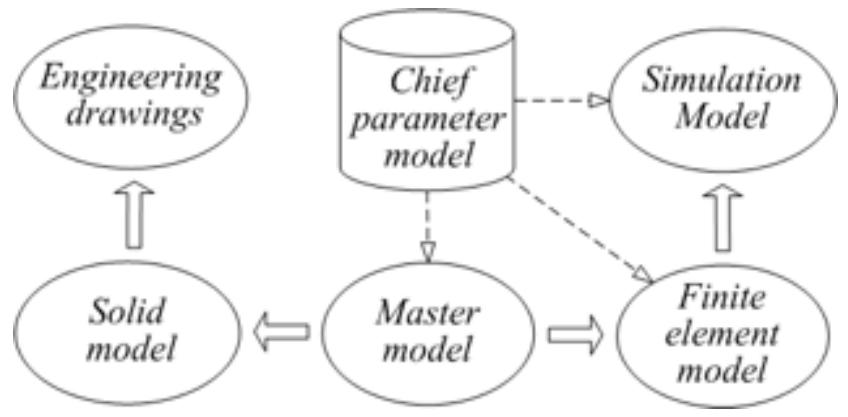

Fig. (1). Synchronous $\mathrm{CAD} / \mathrm{CAE}$ model.

\subsection{Master Model and Solid Model}

Before establishing overall CAD product model, a simplified (or idealized) model named master model is built up to perform engineering analysis and initial evaluation. In summary, master model can be viewed as a sort of unified and shareable integration information model. It can be used directly in FE simulation and serves for 3D solid modeling. Feature-based solid modeling approach is employed to add detail and dimensionality based on the master model till CAD modeling is completed. Meanwhile, engineering drawings are fulfilled through mapping solid model. Hence, solid model and engineering drawings can be changed correspondingly and synchronously by manipulating master model due to feature relationship.

Master model can be represented directly by simplified geometry which is comprised of primary solid, surfaces or wireframes. Solid model may be achieved by master model with the help of feature-based solid modeling approach. For example, (1) a claw part model can be achieved by adding details, e.g. fillet, chamfer and ostiole in a simplified 3D model; (2) with the aid of thickness and amendment algorithm as well as adding details, a welded element model comes into being on the basis of a series of shells; (3) a 3D model of beam truss structure can be generated based on a group of successive wireframes via the operation of sweeping along guilds and adding details.

\subsection{Finite Element Model and Simulation Model}

Finite element analysis has emerged as an important engineering analysis tool and is extensively used to ensure that the design of the model meets the specifications. In order to find out potential structure defect and implement preliminary simulation as early as possible, it is a crucial experience for designers to perform structure analysis in the initial stage of design stage.

Finite element model is dominated by master model because of the CAD-derived polygon geometry for meshing. Besides, nodes, elements, physical and material properties are added into finite element model. Simulation model includes one or more solutions and reusable solver-specific modeling objects from finite element model. In addition, simulation model contains loads, constraints and other boundary conditions. Simulation results can be obtained by solving operations in the basis of FE model and simulation 
model. Thus, finite element model is associated with master model as well as simulation model is related with finite element model. Finite element model and simulation model can refresh synchronously and correspondingly as soon as change happens in master model and a new analysis can be implemented rapidly without duplicated artificial work.

\subsection{Chief Parameter Model}

Chief parameter model can be viewed as a group of parameters such as primary geometric dimensions, design parameters, limits and constraints required to generate preliminary geometry, FE mesh and CAE analysis. In other words, master model, FE model and simulation model can be driven to change by parameters in chief parameter model.

As a centrally organized data structure, The content in chief parameter model is of two categories: (1) geometric parameters, which assist in driving a design concept and conduct crucial dimension in product; (2) Non-geometric functional parameters, including a series of essential physical information associated with FE modeling and simulation, such as material, element size, constrained type and load valve, etc. Besides, chief parameter model is generated in the beginning of design and can be recorded, documented and rationalized. Chief parameter model assists in retaining and monitoring all primary parameters as well as parametric driving modification in master model, finite element model and simulation model with the assistance of knowledgebased tools in CAD systems, e.g. function "expression" in Siemens NX software.

\subsection{File Structure}

In order to contain and operate above models in computer system, file structure is supposed to design and used in parametric parts library. Five kinds of files are involved in this research, namely master file, solid file, FEM file, simulation file and drawing file, which are shown in Fig. (2).

Master file, encapsulating information involved in master model and chief parameter model, is the cornerstone for all the other files in synchronous CAD/CAE modeling process. Master file draws the outline of a part and comprises a group of simple geometry such as wireframes, shells and primary bodies, which can be acted as original objects for CAD and FE modeling.

Solid file is a container to hold detailed and complete feature-based model. With the help of NX/WAVE Geometry Linker, geometries in master file can be coped to corresponding solid file and the resulting geometries is associatively linked to parent geometry. Next, CAD model is further enhanced by adding other features or simply adding more details based on linked geometries until required part is obtained. Thus, changes made in master file can be successfully propagated to solid file. Designers need only copy the requisite geometry meeting design intent.

In the simulation module of NX/Nastran, CAE model is a collection of files that contain all the data required to perform an analysis. The CAE model consists of FEM file and simulation file, which package FE model and simulation model respectively. FEM file contains mesh data, physical and material properties, while simulation file is used to store loads, constraints, solver-specific data, and the solutions that define your analysis. Consistent with the file organization frame, FEM file is a component of the simulation file.

Drawing file is designed to produce and maintain industry standard engineering drawings directly from the 3D model. Drawing sheets created in the NX/Drafting application are fully associative to corresponding solid file. Any changes made to the solid file are automatically reflected in the sheets in drawing file.

Simulation Navigator presents the file relationships and analysis data in CAE model as a graphical, interactive, hierarchical tree. Assembly Navigator is a window that displays context of drawing file, solid file and master file in a hierarchical tree. In part Navigator of drawing file, all sheets are listed in drawing column. Files association in design navigators is shown in Fig. (3).
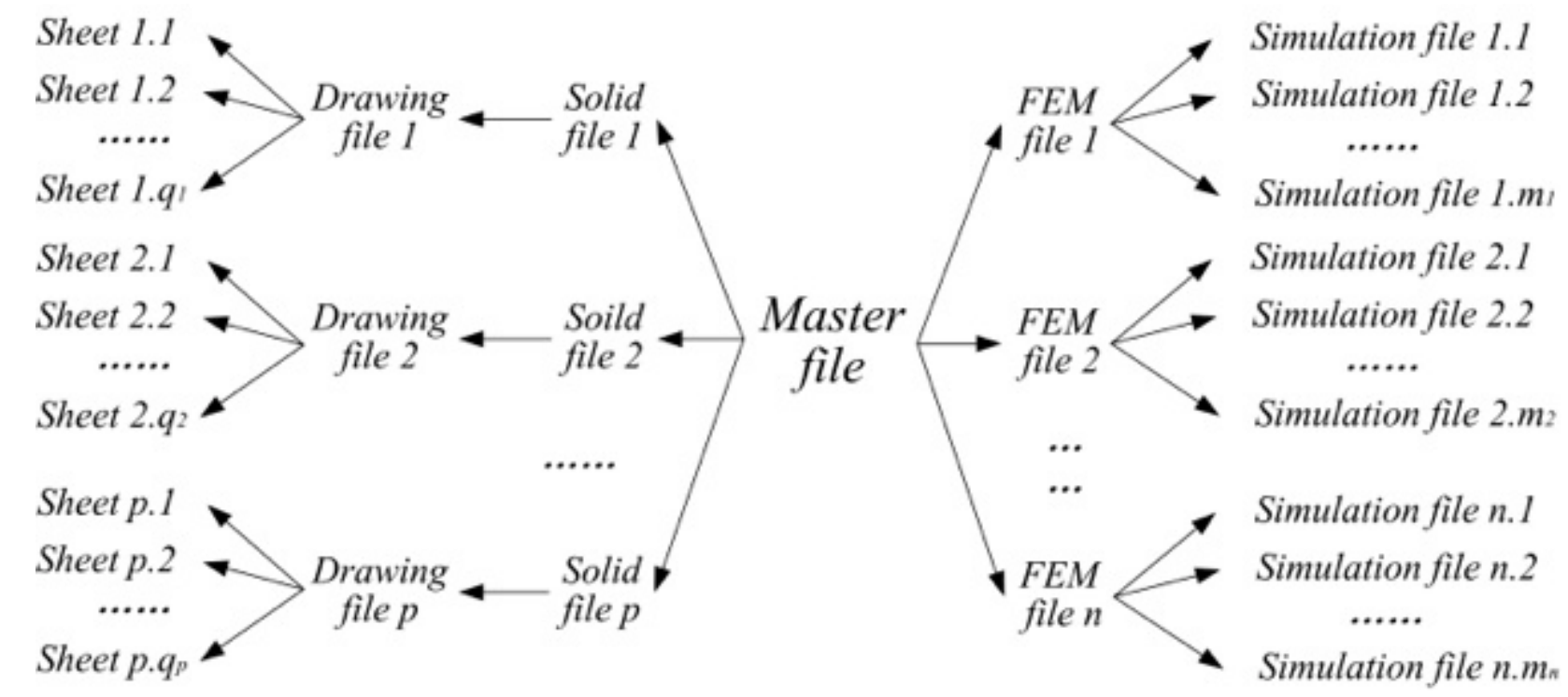

Fig. (2). File structure in parametric parts library. 


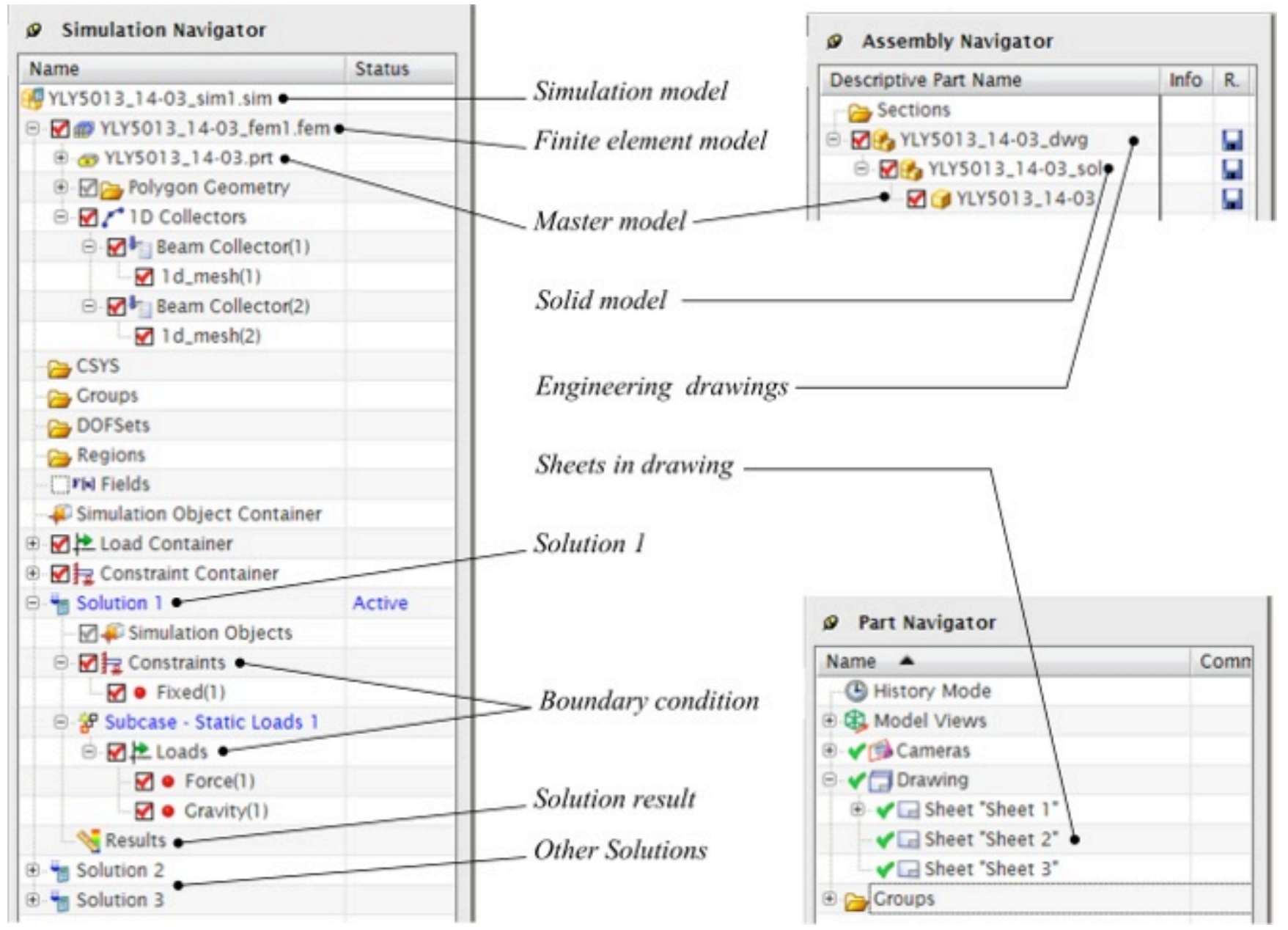

Fig. (3). File association in design navigators.

\section{PARAMETRIC PARTS LIBRARY}

In this research, synchronous $\mathrm{CAD} / \mathrm{CAE}$ modeling method is employed in building parametric parts library. After duplication from part library and parametric modification, parts in parts library can be directly employed to finite element analysis instead of re-modeling in CAE software. If the analysis result is reasonable and acceptable, CAD model and engineering drawings are obtained synchronously with little amendment.

Steps to build parametric parts library are as follows:

Step 1: building up master model, solid model, FE model, simulation model and engineering drawings for parts and defining their relationships. See 2.1 and 2.2

Step 2: extracting chief parameters from mater model, FE model and simulation model into chief parameter model, which is mentioned in section 2.3.

Step 3: classifying parts and setting up a suitable file structure, which helps to supervise all the parts and information in parts library. See section 2.4. Besides, part files (PRT file), concise part introduce (TXT file), part preview picture and parameter chart (JPG file) should be put into designated file path.
Step 4: developing a software package for designers to conveniently search and achieve desired part from parts library with the aid of NX/Open API and C++ language. The package provides the functions of part search from parts library and part parameterization. Figs. $(\mathbf{4}, \mathbf{5})$ provide interfaces for part selection and parameterization respectively.

Steps to use parametric parts library and above package to design a part are as follows:

Step 1: Selecting desired part in parts library with the part search function, see Fig. (4);

Step 2: Duplicating the part to user path, Fig. (4);

Step 3: Change chief parameters of the parts to desirable dimension, see Fig. (5);

Step 4: Updating FE model and simulation model in NX/NASTRAN, then solving and analysis;

Step 5: Solid file and drawing file are just the needed part if analysis result is acceptable;

Step 6: else return to step 3;

Step 7: loop termination till the analysis result is acceptable. 


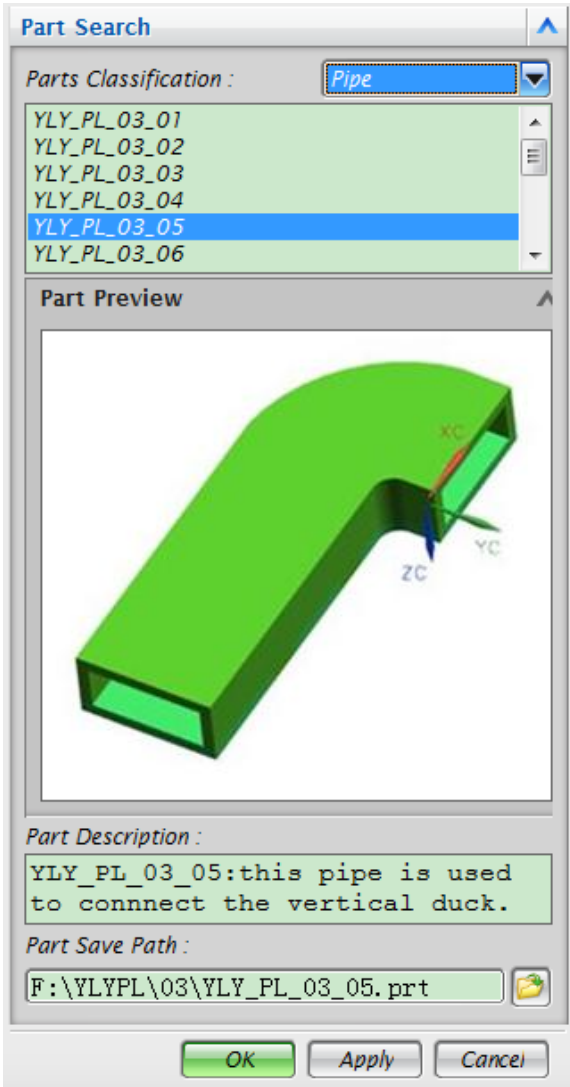

Fig. (4). Interface for part search.

\section{CASE STUDIES}

There are three types of geometry, i.e. plate, primary solid and wireframe, which can be used in master model. Aiming at above distinct applications, three case studies are carried out to clarify synchronous $\mathrm{CAD} / \mathrm{CAE}$ modeling process and change propagation procedure.

The first case is used to illustrate how to build up a synchronous $\mathrm{CAD} / \mathrm{CAE}$ model for thin-wall parts. It means that master model may be in the form of shells. Taking a stamping part as example, it is represented by the feature operation such as extrusion, hole and trimming successively based on a curve. In view of the master model, solid model comes into being via the thicken feature and detailed operation of blending and chamfer. Meanwhile, FE model and simulation model is sequentially fulfilled base on the shells supplied in master model. The modeling process is shown in Fig. (6). The change of parameter (e.g. hole diameter) of this part as well as feature addition (e.g. long hole) in master geometry can be synchronously and automatically propagated to detailed 3D model, FE model and simulation model on account of updating operation. The change propagation process is shown as Fig. (7).

Master model can be delegated by a primary solid body in the second case. Taking a climbing claws part as an example, its master model is implemented by the design feature operation, and then final solid model and FE model are built up as well. Because of applications in distinct working condition, i.e. different boundary condition and constrains, two simulation models and corresponding

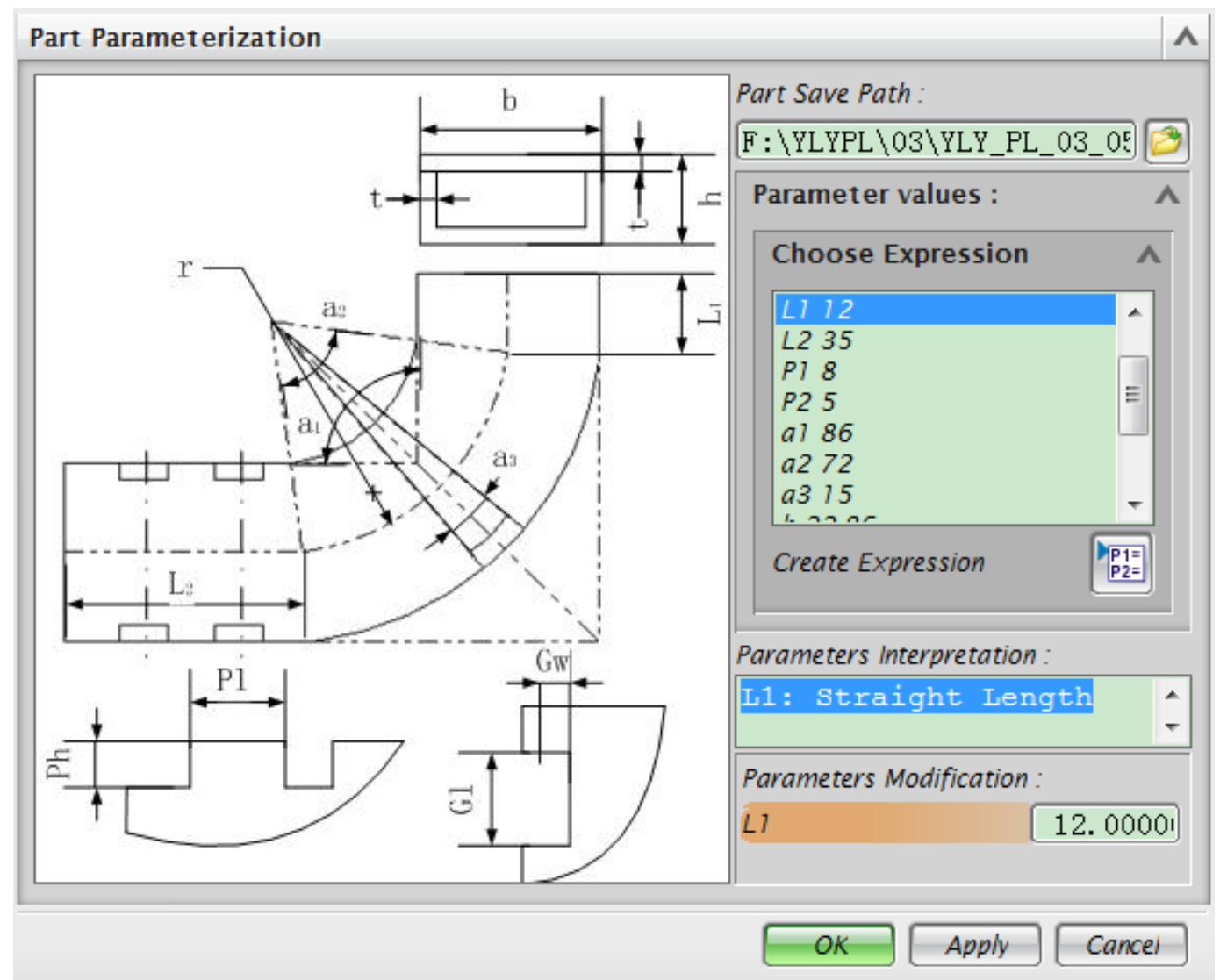

Fig. (5). Interface for part parameterization. 


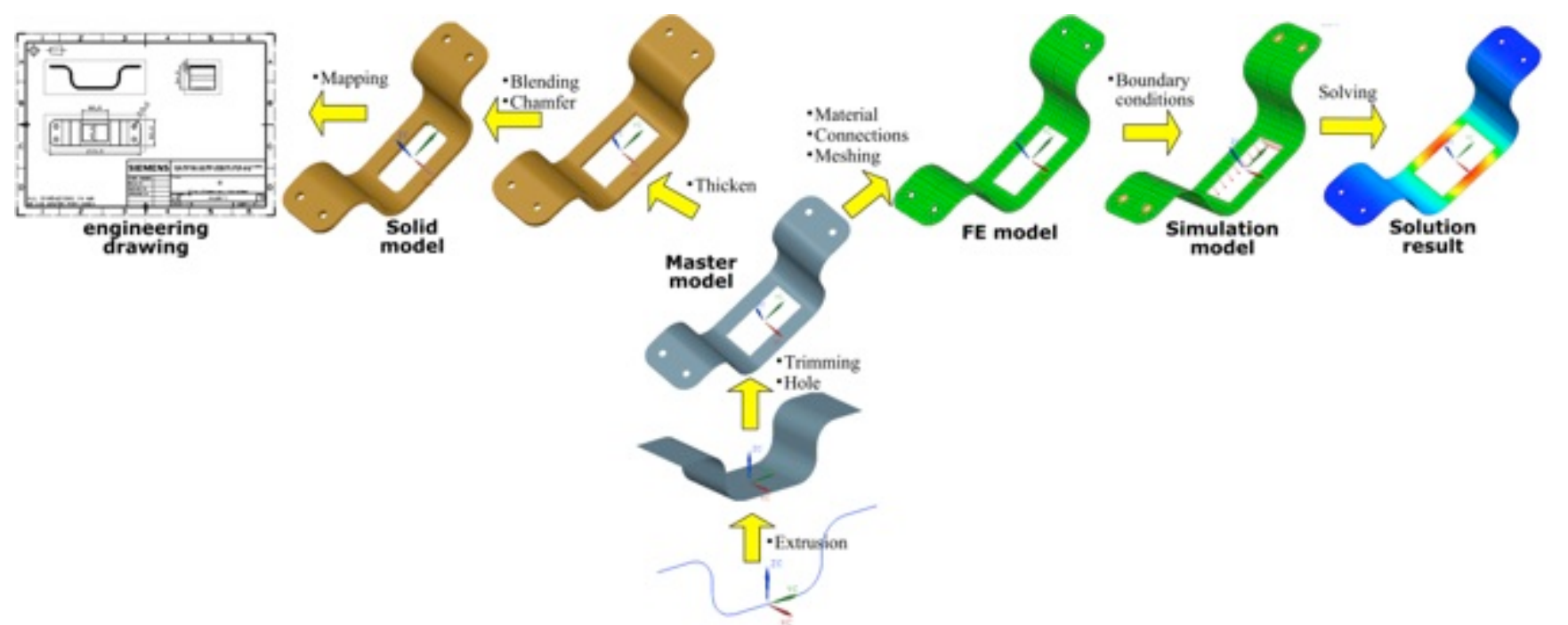

Fig. (6). Modeling process based on shell.

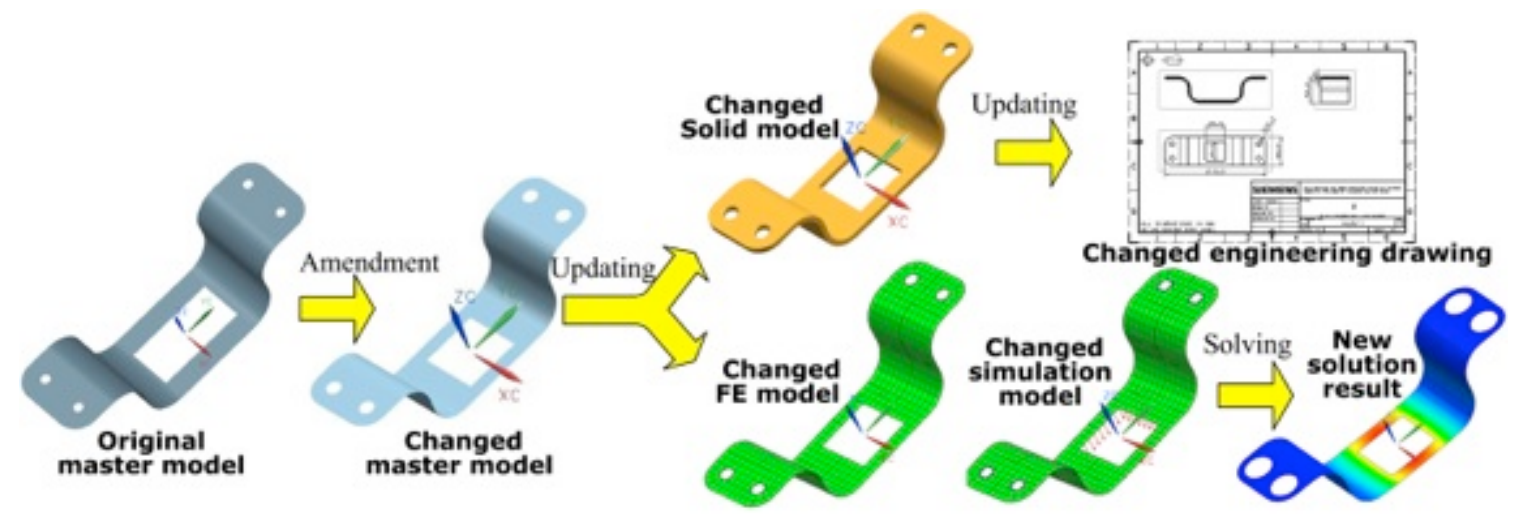

Fig. (7). Change propagation process for stamping part.

solution results are indispensible for this case. The modeling process is shown in Fig. (8).

A kind of part with beam truss structure can be generated in CAD system via the operation of sweeping along successive wireframe, which can be used in master model. Thus, beam element plays a role in FE analysis. It is noted that beam section and number of element should be defined before simulation solving. The modeling process is shown in Fig. (9).

\section{CONCLUSION}

Parts in parts library can be directly employed to FE analysis instead of re-modeling in CAE software. If the analysis result is acceptable, CAD model and engineering drawings are obtained synchronously with little amendment; else parametric amendment and change propagation are sustained.

Master model acts as cornerstone for solid model, FE model and simulation model. It can be represented directly

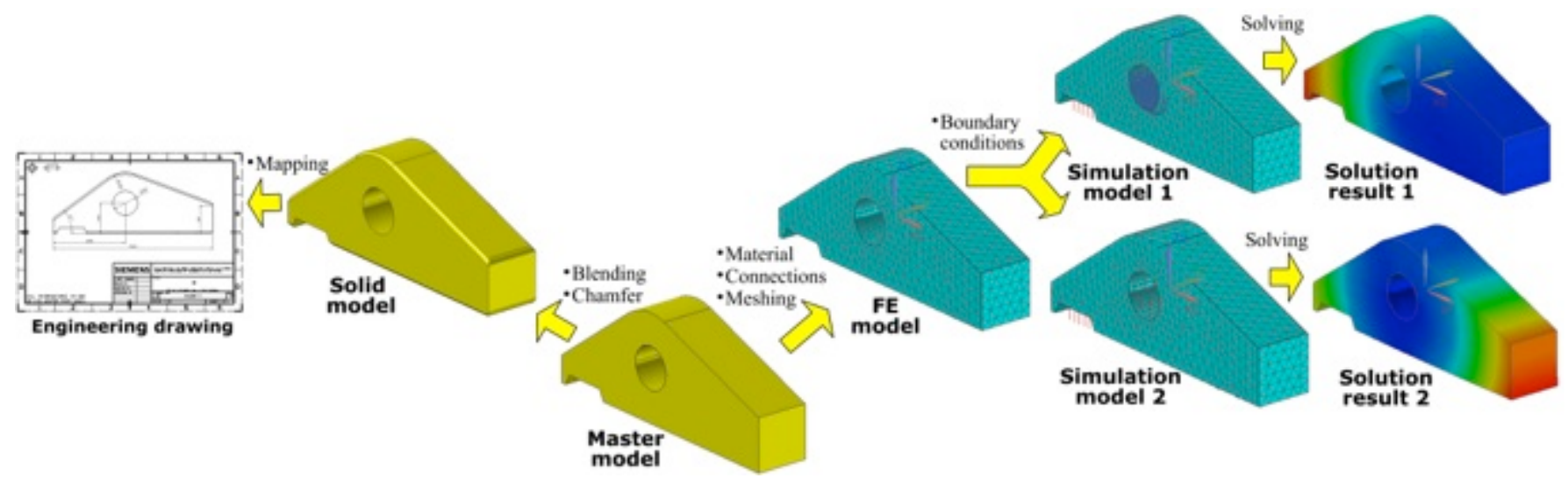

Fig. (8). Modeling process based on primary solid. 


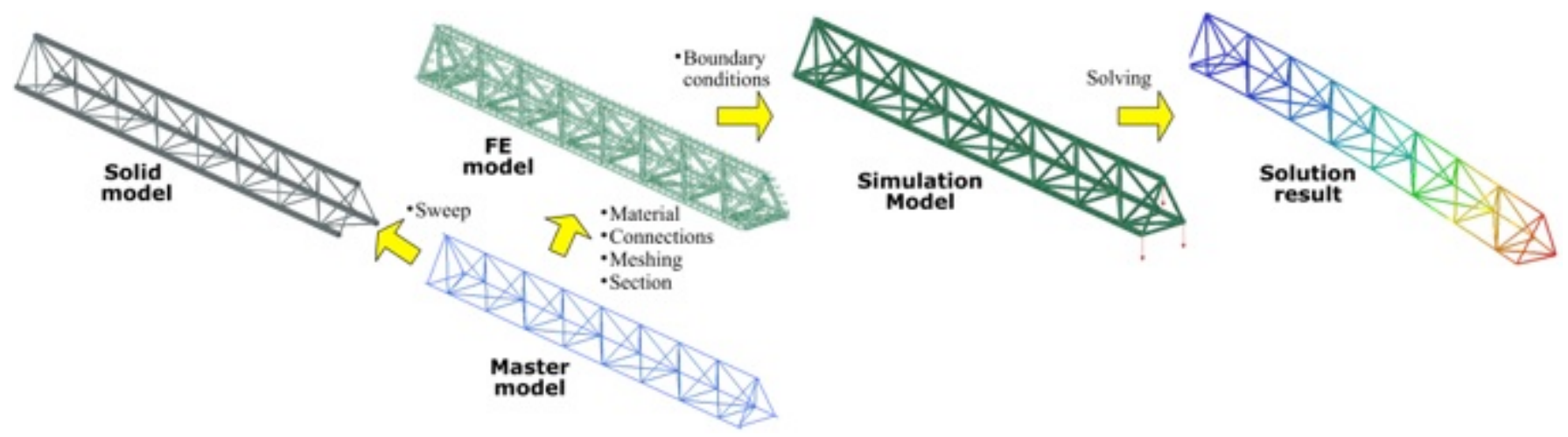

Fig. (9). Modeling process based on wireframe.

by simplified geometry which is comprised of primary solid, surfaces or wireframes. Chief parameter model can revise some other models in parameterization strategy.

A software package developed for designers is helpful to search and achieve desired part from parts library with the aid of NX/Open API and $\mathrm{C}++$ language.

When designers use a parametric parts library to design a part, much duplicated artificial work such as re-modeling and model exchange are dispensable with the help of synchronous CAD/CAE modeling method.

\section{CONFLICT OF INTEREST}

The authors confirm that this article content has no conflict of interest.

\section{ACKNOWLEDGEMENTS}

Declared none.

\section{REFERENCES}

[1] Y. Bo, B. Wang, X.X. Guo, P. Ye, and J. Zhang, "The Development of Brake Three-Dimensional Parts Library Based on CATIA," Applied Mechanics and Materials, vol. 220-223, pp. 2895-2898, 2012.

[2] G. Lu, L. Wang, and S. Lu, "Application and management of modular fixture parts library based on solid works", Advanced Materials Research, vol. 503-504, pp. 103-106, 2012.

[3] G. Lizhi, J. Xiaojun, and H. Qi, "Approach Toward Parametric Design of Typical Parts and Parts-library Based on UG", Applied Mechanics and Materials, vol. 84-85, pp. 8-13, 2011.

[4] M.S. Smit, and W.F. Bronsvoort, "Integration of design and analysis models", Computer-Aided Design and Applications, vol. 6, no. 6, pp. 795-808, 2012.

[5] S. Gao, W. Zhao, and H. Lin, F. Yang, and X. Chen, "Feature suppression based CAD mesh model simplification", ComputerAided Design, vol. 42, no. 12, pp. 1178-1188. 2010.

[6] M. Hamdi, N. Aifaoui, and B. "Louhichi, Idealization of CAD model for a simulation by a finite element method", European Journal of Computational Mechanics, vol. 19, no. 4, pp. 419-429, 2010.
[7] H. Zhu, and C.H. Menq, "B-Rep model simplification by automatic fillet/round suppressing for efficient automatic feature recognition", Computer-Aided Design, vol. 34, no. 2, pp. 109-123, 2002.

[8] A. Thakur, A.G. Banerjee, and S.K. Gupta, "A survey of CAD model simplification techniques for physics-based simulation applications", Computer-Aided Design, vol. 41, no. 2, pp. 65-80. 2009

[9] H. Mounir, A. Nizar and B. Abdelmajid, "CAD model simplification using a removing details and merging faces technique for a FEM simulation", Journal of Mechanical Science and Technology, vol. 26, no. 11, pp. 3539-3548, 2012.

[10] O. Hamri, J.C. Leon, and F. Giannini, et al., "Using CAD models and their semantics to prepare FE simulations", In: Proceedings of the ASME International Design Engineering Technical Conferences and Computers and Information in Engineering Conference - DETC2005, vol. A, pp. 129-138, 2005.

[11] G. Foucault, J.-C. Cuillière , V. François, J.-C. Léon, and R. Maranzana, "Adaptation of CAD model topology for finite element analysis", Computer-Aided Design, vol. 40, no. 2, 2008, pp. 176196.

[12] O. Hamri, J.C. Leon and F. Giannini, and B. Falcidieno, "Software environment for CAD/CAE integration", Advances in Engineering Software, vol. 41, no. 10-11, pp. 1211-1222, 2010.

[13] V. Shapiro, I. Tsukanov and A. Grishin, "Geometric Issues in Computer Aided Design/Computer Aided Engineering Integration", Journal of Computing and Information Science in Engineering, vol.' 11, no. 2, pp. 450-463. 2011.

[14] B.K. Park, and J.J. Kim, "A sharable format for multidisciplinary finite element analysis data", Computer-Aided Design, vol. 44, no. 7, pp. 626-636, 2012.

[15] H.S. Park, and X.P. Dang, "Structural optimization based on CADCAE integration and metamodeling techniques", Computer-Aided Design, vol. 42, no. 10, pp. 889-902, 2010.

[16] T.L. Kenworthy, and C.G. Jensen, "CAD-Centric Dynamic Workflow Generation", Computer-Aided Design and Applications, vol. 6 , no. 5 , pp. 673-683, 2009.

[17] G.P. Gujarathi, and Y.S. Ma, "Parametric CAD/CAE integration using a common data model", Journal of Manufacturing Systems, vol. 30, no. 3SI, pp. 118-132, 2011.

[18] S.H. Lee, "A CAD-CAE integration approach using feature-based multi-re solution and multi-abstraction modeling techniques", Computer-Aided Design, vol. 37, no. 9, pp. 941-955, 2005.

[19] S.H. Lee, "Feature-based non-manifold modeling system to integrate design and analysis of injection molding products", Journal of Mechanical Science and Technology, vol. 23, no. 5, pp. 1331-1341, 2009. 